\title{
Changes in ethical attitudes of clinical laboratory professionals after teaching them ethics using three different methods
}

\author{
Majid Reza Khalajzadeh ${ }^{1}$, Mehrzad Kiani ${ }^{1}$, Fariba Borhani ${ }^{2}$, Shabnam Bazmi ${ }^{1}$, Saeid Nazari Tavakkoli ${ }^{3}$, \\ Mahmoud Abbasi*4
}

Received: 4 Feb 2017

Published: 29 Apr 2019

\begin{abstract}
Background: Ethical attitudes and personal values play a significant role in clinical decision-making; however, they have been given limited attention by professionals in laboratory medicine. Studies suggest that individual attitudes are not static and that professionals learn ethical attitudes through a variety of formal and informal learning methods. This study was conducted to investigate changes in the attitudes of clinical laboratory professionals after teaching them ethics and to compare the results among the 3 groups.

Methods: Four topics were selected in the field of medical laboratory ethics as teaching materials. A questionnaire including 22 items was designed and validated. Teaching sessions for the 3 study groups were held. All 65 clinical laboratory participants completed the questionnaire before and after the classes. Paired $t$ test and ANOVA were used to assess differences among groups.

Results: Significant differences were found in the mean scores of ethical attitudes before and after the educational intervention among the lecture-based teaching group $(\mathrm{p}=0.016)$, problem-based learning group $(\mathrm{p}=0.001)$, and all participants $(\mathrm{p}=0.004)$. However, no significant difference was found between the mean scores before and after the intervention in role-playing group $(\mathrm{p}=0.623)$.

Conclusion: Teaching by lecturing and problem-based learning was more effective to change ethical attitude of the laboratory professionals than the role-playing method. Thus, we suggest the implementation of teaching ethics using these methods to improve the ethical attitude of clinical laboratory professionals.
\end{abstract}

Keywords: Medical ethics, Attitude, Teaching method, Laboratory

Conflicts of Interest: None declared

Funding: None

*This work has been published under CC BY-NC-SA 1.0 license.

Copyright $₫$ Iran University of Medical Sciences

Cite this article as: Khalajzadeh MR, Kiani M, Borhani F, Bazmi Sh, NazariTavakkoli S, Abbasi M. Changes in ethical attitudes of clinical laboratory professionals after teaching them ethics using three different methods. Med J Islam Repub Iran. 2019 (29 Apr);33:36. https://doi.org/10.47176/mjiri.33.36

\section{Introduction}

The primary responsibility of medical laboratory professionals is to provide care to patients and place the welfare of patients above their own needs and desires. Also, they should ensure that each patient receives the highest quality of services and care according to current standards of laboratory practice. Clinical laboratory professionals should exercise sound judgment in set up, performance, and assessment of laboratory testing, and they should also maintain high standards of practice and strict confidentiality of

\footnotetext{
Corresponding author: Dr Mahmoud Abbasi, dr.abbasi@sbmu.ac.ir

1. Department of Medical Ethics, School of Traditional Medicine, Shahid Beheshti University of Medical sciences, Tehran, Iran

2. Medical Ethics and Law Research Center, Medical Surgical Department of Nursing and Midwifery School, Shahid Beheshti University of Medical Sciences, Tehran, Iran

3. Department of Jurisprudence \& the Essentials of the Islamic Law, Faculty of Theology and Islamic Studies, Tehran University, Tehran, Iran

4. Medical Ethics and Law Research Center, Shahid Beheshti University of Medical Sciences, Tehran, Iran
}

patient information and test results. Their ethical attitude safeguards the dignity and privacy of patients and provides accurate information to other health care professionals about the services they provide (1).

Ethical issues have been given limited attention by professionals in laboratory medicine. Unfortunately, many clinical laboratory professionals are either unaware of ethical and legal issues and their importance in the workplace or unable to appropriately deal with these issues (2,

$\uparrow$ What is "already known" in this topic:

Ethical attitude has direct effects on ethical action, and ethics education has an influence on attitude.

$\rightarrow$ What this article adds:

In this study, lecture-based teaching and problem-based learning were more effective than role-playing in changing the ethical attitude after teaching courses for 8 to 10 weeks. 
3).

Personal values and moral attitudes play a major role in clinical decision-making.

Teaching ethics in medical school can enhance competence in medical ethics (4). Ethics education has a significant positive influence on knowledge, moral confidence, moral action, and use of ethics resources (5). Some studies suggest that individual attitudes are not static. Students tend to acquire the attitudes of their professional seniors through a variety of formal and informal learning experiences. Formally, education seeks to instill core ideas and values $(6,7)$.

Traditional methods of medical ethics training offer little help in resolving the ethical dilemmas encountered by health care professionals (8).

Research shows that substantial changes in attitude have developed after years of medical education (9).

With increase in years of medical training, differences appear in students' ethical attitudes (10).

Methods of teaching ethics have been shown to have impacts on educational outcome. Student- and teachercentered approaches are 2 ends of a continuum, and a course can include elements from both extremes or use methods that would be best located between the 2 extremes. In traditional lecture-based method, which is a form of teacher-centered learning, the teacher is solely responsible for what the students are expected to learn, what information should be given, by which methods they should be taught, in what sequence the materials should be presented, and at what pace the course should proceed. The key element in student-centered learning, such as problem-based learning, is that students can learn to determine what they need to know (11).

Problem-based learning is an instructional solution to learning problems, which aims to enhance learning by requiring learners to solve problems. However, roleplaying is a form of simulation and is used as a training method to acquire knowledge, attitude, and skills in a range of disciplines and with learners of different ages $(12,13)$.

Some studies suggest problem-based learning over other methods for teaching ethics $(14,15)$. Noone et al have compared role-playing to traditional lecturing in teaching ethics in areas involving feelings and emotions (16). In other studies, it seems that there are some challenging issues about the methods used to teach ethics. The question is which method of teaching ethics is more effective in changing and improving ethical attitudes of health care professionals.

This study was designed to examine the change in the attitudes of clinical laboratory professionals after teaching them ethics using 3 different methods.

\section{Methods \\ Data}

This was a quasi-experimental study with pretestposttest design. The setting of this study included 347 medical laboratories or hospitals affiliated to Iran University of Medical Sciences, located in western districts of Tehran. Medical laboratory technologists with qualified academic degrees were selected using simple random sampling and a chart of random digits. Exclusion criteria were unqualified academic degree or unrelated discipline. All participants $(n=65)$ signed an informed consent at the beginning of the study. The sample size was calculated statistically and participants were selected for each group. Participants were placed into 3 different groups using a chart of random digits: (1) lecture-based teaching (LBT) $(\mathrm{n}=24)$, (2) problem-based learning $(\mathrm{PBL})(\mathrm{n}=21)$, and (3) role-playing $(\mathrm{RP})(\mathrm{n}=20)$.

\section{Instruments}

1) Based on Iranian Guideline of Ethics in Medical Laboratory, the ethics teaching modules, structured for education and discussion, were selected such that they could cover important moral issues for laboratory technologists. Four topics for ethics teaching modules in medical laboratory were selected as follow: (A) autonomy and informed consent, (B) truth-telling and breaking bad news, (C) privacy and confidentiality, and (D) conflict of interest. Content of the courses was derived from textbooks on medical ethics and supplemented with the researchers' experiences. The module for LBT group was only text for lecture plus 4 example cases. The module for PBL group was a brief text plus 4 example cases and items for discussion. The module for RP group was a brief text plus 4 example cases as scenarios to play. All example cases had similar subjects in 3 groups. The length of teaching session for each topic was 3 hours. Duration of teaching course in this study lasted 8-10 weeks for each group.

2) The researchers used a questionnaire to evaluate the attitudes of participants before and after the teaching sessions. The questionnaire was designed based on the work of Edler et al (7). The Elder's questionnaire contained 25 items for nurses and medical students. Some statements of the questionnaire were selected and modified for laboratory professionals. The 22 -item questionnaire was then sent to 10 expert panels in the field of the study. The questionnaire was validated and to increase validity, researchers changed some items (For example: informed consent should be obtained from the patient before collecting a sample for laboratory test.). Content validity index of the questionnaire used by laboratory technologists reached 0.88 . Reliability of the scores was determined using Cronbach's alpha $(\mathrm{a}=0.80)$. A 5-point response scale (from 1 'not at all' to 5 'strongly agree') was used to explore the extent of agreement with each item. Higher scores indicated a greater degree of acceptable attitudes.

\section{Method of data collection}

Teaching sessions and data collection took place in the early months of 2016. Sessions for the 3 study groups were held only by 1 researcher as a teacher. Each class session started with a short lecture followed by example case study discussions. The educator in LBT group taught the module to the participants by oral and slide presentation. Then, he described all the example cases. In PBL group, the educator first gave a brief oral and slide presentation. Then, he let participants have discussions about example cases. The participants explained their view 
points after reviewing the cases. In RP group, the educator first gave a brief oral and slide presentation. Then, participants played the scenario for each case example while others were observing them, and finally a there was a discussion about scenarios. All participants completed a questionnaire at the beginning and at the end of each course. The written responses were collected anonymously.

\section{Statistical analysis}

Paired t test and ANOVA were used to assess differences among groups before and after teaching courses. Significance level was set at $\mathrm{p}<0.05$ and Statistical Package for Social Sciences (SPSS) was used for data analysis.

\section{Results}

All 65 medical laboratory professionals participated in this study were placed in 3 (LBT, BPL, RP) groups. The mean age of the participants was 38.9 years $(\mathrm{SD}=8.7)$. Of all participants, 21 were male and 44 were female. Work experience of 6 technologists was less than 5 years and 59 had more work experience. Table 1 demonstrates the demographics of the laboratory technologists who participated in class sessions and responded to the questionnaire.

Tables 2 and 3 illustrate the ethical attitude scores before and after the educational intervention, respectively. Statistical calculation by applying analysis of variance separately on data before and after the training revealed no difference in the means of study groups before $(p=0.287)$ and after training $(\mathrm{p}=0.132)$.

Table 4 displays means and standard deviations of the ethical attitude scores before and after the educational intervention in each group and in total. Paired t test was used to analyze the data. Significant differences were observed in the mean scores of ethical attitude before and after the treatment among the LBT group $(p=0.016)$, PBL group $(\mathrm{p}=0.001)$, and all participants $(\mathrm{p}=0.004)$. However, no significant difference was found between the mean scores in RP group $(p=0.623)$. Furthermore, no statistical correlation was observed between ethical attitude score and demographics of the participants.

\section{Discussion}

In this study, the results demonstrated that ethical attitude of clinical laboratory professionals was changed to a higher level after participating in the courses. In most universities of Iran, no courses about ethics have been included in the curriculum for laboratory technologists, so most of the participants in this study had not attended any formal ethics class. The researchers thought that the participants would get low scores at the beginning of the study. However, despite lack of formal ethical education, they obtained a high mean score of ethical attitude before training. This may indicate that they had enough experience of clinical laboratory practice, which has also been shown in demographic data $(8,17)$. Moreover, their high scores may be attributed to the hidden curriculum and role models during their years of practice, which had abled them to provide acceptable work $(6,18)$.

Ethical attitude and a sense of responsibility have direct effects on ethical action and ultimately on the outcome of patient care (19). Seif et al, in their study, demonstrated that medical professionals working in the health care system have an unfavorable theoretical knowledge about medical professionalism in Iran, although this does not indicate that their practices are unethical. This is because sometimes knowledge is not explicit and perhaps it is part of our subconscious. Their study results indicated that one of the prerequisites of possessing a high level of professional ethical attitude and establishing a proper relationship between the medical professionals and the patients is

\begin{tabular}{|c|c|c|c|c|}
\hline Variable & $\begin{array}{c}\text { Lecture-based teaching group } \\
\text { (LBT) }\end{array}$ & $\begin{array}{l}\text { Problem-based learning group } \\
\text { (PBL) }\end{array}$ & $\begin{array}{l}\text { Role-playing group } \\
\text { (RP) }\end{array}$ & $\mathrm{p}$ \\
\hline Age (mean \pm SD), year & $39.6 \pm 5.3$ & $37.4 \pm 7.7$ & $339.8 \pm 7.69 .8 \pm 7.6$ & 0.459 \\
\hline Female & $54.2 \%(n=13)$ & $76.2 \%(n=16)$ & $75 \%(n=15)$ & 0.203 \\
\hline Male & $45.8 \%(n=11)$ & $23.8 \%(n=5)$ & $25 \%(n=5)$ & \\
\hline work experience $>5$ years & $100 \%(n=24)$ & $76.2 \%(n=16)$ & $95 \%(n=19)$ & 0.017 \\
\hline
\end{tabular}

\begin{tabular}{lccc} 
Table 2.Ethical attitude score before training in groups & & & \\
\hline Variable & Mean & Standard deviation & $95 \%$ Confidence interval \\
\hline Lecture-based teaching group (LBT) & 4.18 & 0.62 & $3.93-4.43$ \\
Problem-based learning group (PBL) & 4.17 & 0.51 & 0.287 \\
Role-playing group (RP) & 4.41 & 0.52 & $3.95-4.39$ \\
\hline
\end{tabular}

Table 3.Ethical attitude score after training in groups

\begin{tabular}{lccc}
\hline Variable & Mean & Standard deviation & $95 \%$ Confidence interval \\
\hline Lecture-based teaching group (LBT) & 4.52 & 0.42 & $4.35-4.70$ \\
Problem-based learning group (PBL) & 4.63 & 0.12 & 0.132 \\
Role-playing group (RP) & 4.37 & 0.54 & $4.54-4.73$ \\
\hline
\end{tabular}

Table 4.Ethical attitude score before and after training in groups

\begin{tabular}{lcc}
\hline Variable & Before education & After education \\
\hline Lecture-based teaching group (LBT) & $4.18 \pm 0.62$ & $4.52 \pm 0.42$ \\
Problem-based learning group (PBL) & $4.17 \pm 0.51$ & $4.63 \pm 0.21$ \\
Role-playing group (RP) & $4.41 \pm 0.52$ & $4.37 \pm 0.54$ \\
Total & $4.25 \pm 0.56$ & 0.001 \\
\hline
\end{tabular}


to have a proper knowledge of this concept. Improving performances and behaviors in medical professions requires adequate training and assessment of the levels of professionalism achieved (20). Correct perception about an ethical issue would be gained when knowledge is sufficient, which would lead to a positive attitude towards the issue (21).

Statistical data revealed that members of the 3 study groups were homogenously distributed. The mean score of ethical attitude in LBT and PBL groups and in total participants were increased significantly, but there was minimum change in RP group. In this study, lecture-based teaching was more effective than role-playing method. This may be explained by unfamiliarity of participants with role-playing method, bad design of the scenarios, ineffectiveness of the method on attitude, or other reasons.

Heidari et al has quoted, "the method of teaching medical ethics in Iran is still lecture-based in most medical schools."

However, a suitable teaching program would be based on those new methods with student participation and active learning (14).

In this study, the duration of teaching courses was 8-10 weeks. Although these courses have considerable effects on outcome, here the medium duration of courses seems to be optimal.

In a meta-analysis, Schlaefli et al divided the researches on moral development according to the program duration into 3 groups: (1) programs with short duration (0-3 weeks), (2) medium duration (4-12 weeks), and (4) long duration (13-28 weeks). Short duration programs have the smallest effect, long-term programs have medium effect, and medium duration programs show the largest effect. Thus, there is no linear relationship between duration of a teaching program and its effect. Therefore, longer duration of a teaching program does not guarantee increased moral development (11).

\section{Conclusion}

Medium duration course of teaching ethics to health care professionals, such as clinical laboratory technologists, is able to change ethical attitude. Teaching by lecturing and problem-based learning is more effective to change ethical attitude than the role-playing method. Thus, we suggest implementation of teaching ethics by these methods to improve the ethical attitude of clinical laboratory professionals. We also recommend additional research in the field of teaching ethics for other clinical professionals.

\section{Limitations}

Short duration of educational course, method of education, and the instrument (questionnaire) were some of the limitations of this study which should be considered in upcoming studies.

\section{Acknowledgments}

Gratitude is expressed to the School of Traditional Medicine in ShahidBeheshti University of Medical Sciences for approving this study. Also, thanks go to Dr. Mozaffari,
Dr. Paydar, Dr. Almasi, Dr. Akef, and Ebad Nezami.

\section{Conflict of interest}

The authors declare that there is no conflict of interest. The research was approved by IRB of Shahid Beheshti University of Medical Sciences.

\section{References}

1.Plebani M, Lippi G. Is laboratory medicine a dying profession? Blessed are those who have not seen and yet have believed. Clin Biochem. 2010;43(12):939-41.

2. Wijeratne N, Benatar SR. Ethical issues in laboratory medicine. BMJ Publishing Group Ltd and Association of Clinical Pathologists; 2010.

3. Arora D, Arora B. Ethics in laboratory medicine. Ind J Med Microbiol. 2007;25(3):179.

4. Wandrowski J, Schuster T, Strube W, Steger F. Medical ethical knowledge and moral attitudes among physicians in Bavaria. Dtsch Arztebl Int. 2012;109(8):141-7.

5. Grady C, Danis M, Soeken KL, O'Donnell P, Taylor C, Farrar A, et al. Does ethics education influence the moral action of practicing nurses and social workers? Am J Bioeth. 2008;8(4):4-11.

6. Borhani F, Alhani F, Mohammadi E, Abbaszadeh A. Professional Ethical Competence in nursing: the role of nursing instructors. J Med Ethics Hist Med. 2010;3:3.

7. Elder R, Price J, Williams G. Differences in ethical attitudes between registered nurses and medical students. Nurs Ethics. 2003;10(2):14964.

8. Hariharan S, Jonnalagadda R, Walrond E, Moseley H. Knowledge, attitudes and practice of healthcare ethics and law among doctors and nurses in Barbados. BMC Med Ethics. 2006;7(1):7.

9. Lynoe N, Löfmark R, Thulesius H. Teaching medical ethics: what is the impact of role models? Some experiences from Swedish medical schools. J Med Ethics. 2008;34(4):315-6.

10.Gruber PC, Gomersall CD, Joynt GM, Lee A, Tang PYG, Young AS, et al. Changes in medical students' attitudes towards end-of-life decisions across different years of medical training. J General Int Med. 2008;23(10):1608-14.

11.Clarkeburn $\mathrm{H}$. How to teach science ethics: University of Glasgow; 2000.

12.Savery JR. Overview of problem-based learning: Definitions and distinctions. Essential readings in problem-based learning: Exploring and extending the legacy of Howard S Barrows. 2015:5-15.

13. Nestel D, Tierney T. Role-play for medical students learning about communication: guidelines for maximising benefits. BMC Med Educ. 2007;7(1):3.

14.Heidari A, Adeli SH, Taziki SA, Akbari V, Ghadir MR, MoosaviMovahhed SM, et al. Teaching medical ethics: problem-based learning or small group discussion? J Med Eth Hist Med. 2013;6.

15.Jones NL, Peiffer AM, Lambros A, Guthold M, Johnson AD, Tytell $\mathrm{M}$, et al. Developing a problem-based learning (PBL) curriculum for professionalism and scientific integrity training for biomedical graduate students. J Med Ethics. 2010;36(10):614-9.

16. Noone PH, Sharma SR, Khan F, Raviraj K, Shobhana S. Use of role play in undergraduate teaching of ethics-An experience. J Forens Legal Med. 2013;20(3):136-8.

17.Nilstun T, Cuttini M, Saracci R. Teaching medical ethics to experienced staff: participants, teachers and method. J Med Ethics. 2001;27(6):409-12.

18.Perry RB. Role modeling excellence in clinical nursing practice. Nurse Educ Pract. 2009;9(1):36-44.

19.Moosavi S, Borhani F, Mohsenpour M. Ethical attitudes of nursing students at Shahid Beheshti University of Medical Sciences, Iran. Ind J Med Ethics. 2016:1.

20.Seif-Farshad M, Bazmi S, Amiri F, Fattahi F, Kiani M. Knowledge of medical professionalism in medical students and physicians at Shahid Beheshti University of Medical Sciences and affiliated hospitalsIran. Medicine. 2016;95(45).

21.Motamed-Jahromi M, Abbaszadeh A, Borhani F, Zaher H. Iranian nurses' attitudes and perception towards patient advocacy. ISRN Nurs. $2012 ; 2012$ 